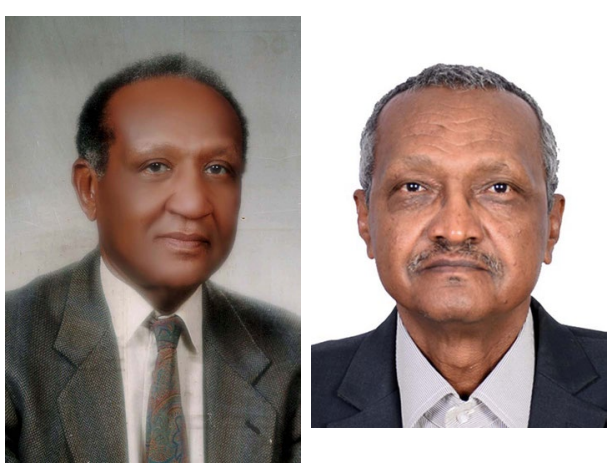

Credit: Images courtesy of Mustafa Khogali (left) and Maowia M. Mukhtar (right)

udan is a low-income country with a diverse ethnic population. It is bordered by seven countries, with high movement across the borders. According to UN data, the Sudanese population is $\sim 44$ million people. The majority of the population is rural based, but during the last decades there has been massive migration to urban areas, resulting in the spread of infectious and endemic diseases, for example, malaria, pulmonary tuberculosis and typhoid fever, along with recurrent outbreaks of arboviruses, exhausting the weak health system.

Sudan was not prepared to manage and control the emerging COVID-19 pandemic. Diagnosis of COVID-19 was slow and was based on CT scans and PCR with reverse transcription (RT-PCR). The RT-PCR tests were initially centralized in the national health laboratory, slowing the diagnosis and identification of individuals who were virus-positive, which limited tracing of contacts. It took the health authorities more than 6 months to decentralize the diagnosis and screening of individuals. Several public and private health laboratories were later licensed to conduct diagnostic RT-PCR tests. However, access to such private testing was and still is unaffordable for most of the population.

The first case of COVID-19 was diagnosed on 12 March 2020, in a 50 -year-old Sudanese male who arrived via air from United Arab Emirates. He developed respiratory symptoms, was admitted to hospital and died 3 days later with a positive COVID-19 RT-PCR test. This raised the alarm for the arrival of SARS-CoV-2 in Sudan.

Data on community transmission of COVID-19 was limited due to the small-scale serology and molecular surveys conducted in Khartoum State. The first community transmission occurred on 28/29 March 2020. Accordingly, the health

\title{
The accelerating COVID-19 epidemic in Sudan
}

\author{
Mukhtar and Khogali discuss the many challenges Sudan has faced in \\ coping with the COVID-19 pandemic.
}

authorities recommended the use of face masks and social distancing, although these were not supported by government legislation. Khartoum State (KS), including the capital, has a population of 8 million people ( $19 \%$ of the total population). KS has been the epicenter of the epidemic since it began, always housing more than $70 \%$ of all COVID-19 cases. On April 12021 , a total of 30,404 cases was reported in Sudan, 21,728 (72\%) of which were in KS. This represents an incidence rate per 100,000 of 259 in KS as compared to a range in the incidence rate per 100,000 of $1-70$ among the other 17 states. This figure is probably due to the large population and social behavior of those living in $\mathrm{KS}$ as well as the inequity in accessing the diagnostic test in other states.

On 16 March 2020, the health authorities closed the airport and the other points of entry. However, two flights were allowed to land at Khartoum airport on the 26th and 28th, returning Sudanese citizens who had been stranded abroad and evacuating expatriates from Sudan. Travelers on the second flight were not effectively tested and isolated, which increased the risk of importing the virus into the country. A curfew was introduced on 16 March. Following a surge in COVID-19 infections, a complete lockdown was imposed during the period of 24 April to 8 July 2020.

The lockdown was resisted by the community because of increased economic hardship for families. Moreover, the decision to lockdown was not based on sufficient data on the extent of the community transmission, and areas that required lockdown were not accurately defined. The government offices were closed, with the exception of the essential services, such as banks and emergency health services. However, the lockdown was not effectively implemented due to lack of proper law enforcement.

An effective tracing system is yet to be implemented. Tracing was based on voluntary data provided by the patient and/ or through contact tracing using telephone calls and home visits. The ineffective tracing slowed early identification and isolation of infected individuals and contributed to the occurrence of the second wave during the period of November 2020 to January
2021. The second wave was much more severe, for reasons that are currently unclear. Virus genome sequencing is currently in progress and may shed some light on the epidemiology, pathophysiology and prevalence of virus variants. The second wave affected all age groups with high mortalities, reaching 20\% among patients in the intensive care unit. To date, more than 30,000 patients with COVID-19 have been diagnosed, with an overall case fatality rate of $6.9 \%$.

The pandemic has had an impact on health services. Most of the public and private clinics were closed due to the high infection and mortality rates among healthcare providers. Patients with chronic diseases and those who required emergency medical interventions were obviously affected. COVID-19 isolation centers and intensive care services were overwhelmed due to the lack of sufficient beds and ventilators. While the World Health Organization had issued treatment guidelines, adopted by the Ministry of Health, the lack of public awareness and confidence led many patients to use traditional local herbal medicines.

Sudan received the Oxford-AstraZeneca vaccine in early March 2021 through the COVAX Program and started the vaccination campaign on 9 March. The priority target groups for vaccination are healthcare workers at all levels of the health service, persons aged 60 years and more and patients with comorbidities aged 45 years and over. Currently, there is no time frame for the expansion of vaccine eligibility to those younger than 60 . To facilitate this, more vaccine supply is desperately needed. The timing for the second dose will depend on the availability of the vaccine as scheduled by COVAX/AstraZeneca.

Sudan has faced many challenges in managing the pandemic due to the lack of accurate and rapid diagnosis, screening to identify and isolate infected individuals, a proper tracing system, and data on community transmission and vaccine supply. We hope to overcome these challenges during the third wave, which started in March 2021 and is currently ongoing. If nothing changes, Sudan, like 


\section{WORLD VIEW I SERIES}

many other countries, could be severely crippled by the pandemic.

Maowia M. Mukhtar (D) 1,2,4凶 and Mustafa Khogali 3,4

${ }^{I}$ Institute of Endemic Diseases, University of
Khartoum, Khartoum, Sudan. ${ }^{2} T I B A-S u d a n$

$\square \quad$ Project, Bioscience Research Institute, Ibn Sina University, Khartoum, Sudan. ${ }^{3}$ School of Medicine, Ahfad University for Women, Omdurman, Sudan. ${ }^{4}$ These authors contributed equally: Maowia M. Mukhtar and
Mustafa Khogali.

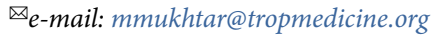

Published online: 25 May 2021

https://doi.org/10.1038/s41590-021-00950-0 